\title{
El desempeño económico financiero y responsabilidad social corporativa Petrobrás versus Repsol
}

\author{
Miguel Lopes \\ de Oliveira Filho \\ Universidade Federal de \\ Pernambuco.miguel@ufpe.br

\section{José Mariano Moneva}

\section{Abadía} \\ Universidad de Zaragoza \\ jmmoneva@unizar.es
}

\section{Resumen}

Los cambios en el ambiente institucional, como resultado del proceso de integración de los mercados y las consecuentes alteraciones en el comportamiento de los stakeholders, son uno de los más importantes vectores de cambios en las conductas de las empresas. En ese contexto, además de sus rentabilidades, esas empresas se preocupan por sus actitudes en los factores sociales y ambientales. Así, la responsabilidad social corporativa (RSC) es vista como una estrategia más para mantener o aumentar su rentabilidad y potenciar el desarrollo empresarial. La finalidad principal de este estudio es la verificación de la relación existente entre la responsabilidad social y el desempeño económico-financiero de dos empresas dedicadas al negocio del petróleo, gas y biocombustibles. La investigación se basa en una comparación de las prácticas socioambientales desarrolladas por Petrobrás, S.A. (Brasil) y Repsol, S.A. (España). Con objetivo de comprobar la relación entre el desempeño socioambiental y el económicofinanciero se ha llevado a cabo un análisis de regresión lineal.

Palabras clave: responsabilidad social corporativa, desempeño económico, desempeño financiero, expectativas de los stakeholder 


\title{
The economic and financial performance and corporate social responsibility: Petrobras versus Repsol
}

\begin{abstract}
Alterations in the institutional environment, as a result of the markets integration process and the consequent alterations in stakeholders' conduct are one of the important vectors of changes in companies' behavior. In that context, in addition to their yields those companies happen to worry about their attitudes in relation to social and environmental factors. Thus, the corporate social responsibility (CSR) is seen as one more strategy to maintain or increase their income and to harness the enterprise development. The main purpose of this study is the verification of the existing relation between Social Responsibility and the economic and financial performance of two companies in the business of Petroleum, Gas and Bio-combustibles. The investigation was based on a comparison of social-environmental practices developed by Petrobras S/A (Brazil) and Repsol S/A (Spain). For the purpose of verification of the relation between social-environmental performance and the economicfinancial one, a linear regression analysis was carried out.
\end{abstract}

Keywords: corporative social responsibility, economic performance, financial performance, stakeholder's expectations

\section{Introducción}

En los últimos años, el papel socio-económico desempeñado por las empresas es un motivo de gran atención por parte de los grupos de interés a la hora de efectuar sus inversiones financieras y comerciales. Con estas actitudes, las empresas se han visto obligadas a replantearse sus posiciones y efectuar severos cambios en sus conductas social y ambiental. De esta manera, las empresas prestan mayor atención a la hora de incluir la responsabilidad social corporativa (en adelante RSC) en sus estrategias, de forma que logran unificar los intereses socio-ambientales con los económicos-financieros (González, 2004). Este enfoque, relativamente moderno, cuyos orígenes se pueden hallar desde los años setenta (Moneva y Llenna, 1996; Moneva, 2005), ha experimentado una aceleración vertiginosa, tanto en la acción como en la reflexión, por parte de los grupos de interés y, consecuentemente, también por las empresas.

La relación entre la RSC y el desempeño financiero viene siendo objeto de estudio 
desde hace muchos años. Sin embargo, cabe destacar que desde el inicio de la década de los sesenta (Toro, 2006) el significado del término en cuestión ha cambiado de una forma bastante radical. Algunos investigadores (Waddock y Graves, 1997; Stanwick y Stanwick, 1998; Ruf et al., 2001; Mahoney y Roberts, 2007; Zhang y Stern, 2007) concluyen que la RSC está directamente relacionada con la estrategia económica de la empresa e influye positivamente en la rentabilidad financiera creando de esta forma valor a la empresa.

En España, el primer informe sobre responsabilidad social surgió al final de los años setenta (Seara, 2004) con la publicación del balance social del Banco de Bilbao (Fernández Izquierdo et al., 2005; Moneva y Llenna, 1996), el cual tuvo una notable repercusión en el mundo empresarial español; pero las empresas empiezan a utilizar informes de sostenibilidad y a divulgar información en materia de RSC a finales de los años noventa (Sánchez, 2004).

En Brasil, la RSC obtuvo notoriedad a mediados de la década de los noventa, con el lanzamiento de la campaña por la divulgación del balance social, por el sociólogo Herbert de Souza, quien creía en la construcción de una sociedad más justa e igualitaria con respecto a los derechos fundamentales y al ejercicio de la ciudadanía, defendiendo la participación de los empresarios en el desarrollo social (IBASE, 2008).

En Brasil no existe normativa ambiental aplicada al reconocimiento, valoración e información de los aspectos medioambientales en los informes anuales. Sin embargo, hay un proyecto de ley en tramitación en el poder legislativo que propone la obligatoriedad del balance social. Actualmente, las empresas brasileñas que cotizan en bolsa publican un informe específico disponible en la página web de la Comisión de Valores Mobiliarios (CVM, 2008), donde presentan algunas cuestiones ambientales.

La mayor parte de la información sobre las actividades sociales de las empresas brasileñas se publican de manera voluntaria, pero la simple divulgación — por parte de las empresas- de noticias en sus páginas web sobre la existencia de políticas y programas sociales, sin la incorporación de las mismas en informes anuales, no demuestra el compromiso ni la dimensión de las acciones sociales de la organización en relación con los diferentes grupos de interés (Milani Filho, 2008). En investigaciones de la Confederación Nacional de la Industria (CNI) y del Instituto 
Brasileño de Opinión Pública y Estadística (IBOPE) destaca que el 68\% de los consumidores brasileños estarían dispuestos a pagar más por un producto que no agrediese al medioambiente (Tachizawa, 2004).

En las últimas décadas se ha debatido mucho sobre la relación entre los intereses económicos empresariales y sus actuaciones externas en relación con las prácticas sociales y ambientales. Esos intereses, potencialmente por regla general, pueden causar muchos impactos ambientales y sociales negativos como contaminación, deforestación y mala distribución de renta.

La creciente consciencia social en cuanto a los problemas socio-ambientales, como el calentamiento global, la escasez de recursos hídricos y los conflictos étnicos, está llevando a las empresas a reconsiderar sus prácticas de gestión, buscando entender hasta qué punto esos fenómenos pueden afectar a sus negocios. Actualmente es bastante significativa la cantidad de empresas que incorporan en sus estrategias aspectos sociales y ambientales. Con base en esos argumentos, y teniendo en cuenta los proyectos de RSC desarrollados por Petrobrás, S.A. y Repsol, S.A., esta investigación procura responder la siguiente pregunta: ¿Hasta qué punto las inversiones sociales y ambientales, desarrolladas por Petrobrás y Repsol, influyen en sus desempeños económico-financieros? La investigación tiene como objetivo principal identificar si las variables ambientales y sociales, desarrolladas por Petrobrás y Repsol, están relacionadas con sus desempeños económico-financieros.

La RSC se viene consolidando como un tema de creciente interés e importancia entre la comunidad académica y la comunidad empresarial. En este sentido, es conveniente decir que la responsabilidad social puede ser comprendida en un contexto más amplio, incluido en la noción de sostenibilidad corporativa; de igual forma, prioriza el compromiso con el desarrollo sostenible basada en tres dimensiones de sostenibilidad: la social, la ambiental y la económica (GRI, 2008).

Diversos grupos de interés son cada día más incisivos a la hora de demandar informaciones y rendición de cuentas al respeto de las prácticas ambientales y sociales de las empresas. Los inversionistas buscan evidencias de buenas prácticas de gobierno corporativo y de gestión de riesgo. Los consumidores cuestionan a las empresas sobre la forma, los componentes y el destino de los envases de los productos. Los empleados buscan empresas que valoran el capital humano, asuman responsabilidades sociales y ambientales y presenten cuentas a la sociedad sobre 
sus acciones corporativas. Los gobiernos y la sociedad civil aumentan la presión sobre las compañías para que informen sobre los resultados obtenidos en los campos social y ambiental (Lewis, 2003).

\section{Referencial teórico}

El Instituto Ethos (2007) reconoce la RSC como una forma de conducir los negocios que vuelve a la empresa compañera y corresponsable por el desarrollo social y ambiental. La empresa socialmente responsable es aquella que posee la capacidad de escuchar los intereses de las diferentes partes (accionistas, empleados, prestadores de servicios, proveedores, consumidores, comunidad, gobierno y medio ambiente) y conseguir incorporarlos a la planificación y estrategia de sus actividades buscando atender las demandas de todos, no sólo de los accionistas o propietarios.

Para que una empresa pueda ser considerada socialmente responsable no es suficiente con que apoye el desarrollo de la comunidad y preserve el medioambiente, sino que también es necesario que invierta en el bienestar de sus empleados y dependientes, además de promover comunicaciones transparentes, ofrecer rentabilidad a los accionistas, asegurar sinergias con sus compañeros y garantizar la satisfacción de sus clientes y/o consumidores.

El desempeño empresarial depende muchas veces de la utilización y/o consumo de recursos naturales, sean renovables o no, que no son de su propiedad y sí de la sociedad. La empresa al utilizar esos recursos en beneficio propio está contrayendo una deuda social con la humanidad, pues aquéllos constituyen un patrimonio de la sociedad; a cambio, las empresas deberán contribuir a la solución de los problemas sociales y ambientales.

En este contexto, las empresas no dejan de incluir las ganancias como objetivo; sin embargo, al contrario de priorizar la maximización de beneficios a corto plazo, las organizaciones deberían buscar beneficios a largo plazo, obedecer las leyes, considerar el impacto no mercadotécnico de sus decisiones y buscar formas de mejorar la sociedad con una actuación orientada a la RSC (Ethos, 2007).

En la práctica, esos principios consisten en, por ejemplo, fabricar productos ambientalmente responsables de manera que mejore su posición competitiva, aprovecharse de las oportunidades propiciadas por requisitos legales para crear productos 
que puedan ofrecer una contribución especial a la sociedad, suplir necesidades sociales comercializando productos que beneficien a grupos específicos como minusválidos y minorías, utilizar voluntariamente recursos de la organización para ayudar a solucionar problemas sociales y ambientales.

Según Mcwilliams y Siegel (2001), la responsabilidad corporativa, como estrategia de diferenciación, es usada para crear nuevas demandas y obtener un precio premium para un producto o servicio ya existente. Algunos consumidores quieren que los productos que compran presenten algunos atributos de responsabilidad social (innovación de productos); otros valoran los productos que son fabricados de forma responsable (innovación de proceso).

En el ejercicio de la responsabilidad social la empresa actúa en dos dimensiones: enfocado al público-interno, es decir, empleados y dependientes, configurando la responsabilidad social interna; y enfocado al público externo, es decir, comunidad y sociedad, cumpliendo con su responsabilidad social externa.

Es posible visualizar algunas acciones de la gestión de la empresa que se caracterizan como cumplimiento de su responsabilidad social interna y externa. Como interna se pueden citar los programas de contratación, selección, entrenamiento, manutención de personal y participación en los resultados, realizados por las empresas a favor de sus empleados. Como acciones de responsabilidad social externas, introducidas en la estrategia de las empresas, es posible observar las acciones sociales que benefician a la comunidad, las cuales pueden ser realizadas a través de donaciones de productos, equipamientos y materiales por regla general, o traspaso de recursos para órganos públicos y ONG, aplicaciones de recursos en actividades de preservación del medio ambiente, entre otros.

El consumo es, junto con la inversión, una de las palancas más efectivas para impulsar a las empresas hacia la adopción de políticas y sistemas de RSC. Los consumidores pueden, a través de sus compras, premiar o castigar a las empresas en función de su responsabilidad social, convirtiendo así sus actos de consumo en un verdadero acto de transformación social. Para ello es fundamental que esos consumidores dispongan de información actualizada y fiable (Fundación Ecología y Desarrollo, 2006).

Diversos investigadores intentan identificar y clasificar las razones que están llevando a las empresas a buscar una actuación socialmente más responsable. Una de 
las más destacadas es el aumento de presión por parte de la sociedad (Morimoto et $a l ., 2005)$. Además de la presión ejercida por las organizaciones no gubernamentales, la actuación de la empresa está siendo rebatida por comunidades, gobiernos, empleados y hasta por los propios accionistas reunidos en grupos de inversionistas socialmente responsables. El marketing institucional promovido por la buena reputación, producto de un posicionamiento socialmente más justo, es otra motivación importante porque las acciones judiciales movidas por organizaciones no gubernamentales pueden implicar un serio perjuicio para la imagen de las empresas en el mercado.

Morimoto et al. (2005) destacan que la RSC puede ser una herramienta para legitimar la actuación de la empresa frente a sus diversos interesados por las actividades sociales de la empresa, pues más comunicación y transparencia pueden ayudar a desarrollar una imagen positiva de la organización.

Knox et al. (2005), así como Payne y Raiborn (2001) afirman que las empresas deberían preocuparse por el desarrollo sostenible por tres motivos: por la propia continuidad de la existencia de las empresas y de la sociedad, por el desarrollo de la sostenibilidad como competencia central para obtención de ventajas competitiva y para mejorar su reputación.

En un estudio desarrollado por White y Kiernan (2004) se demostró cómo la buena gestión ambiental puede interferir en las perspectivas económicas de la empresa, mejorando la rentabilidad financiera. Se realizaron 15 estudios comparados en once empresas de cuatro sectores productivos. En el $85 \%$ de los casos estudiados se encontró una correlación positiva entre la buena gestión ambiental y el desempeño financiero.

\section{La responsabilidad social corporativa y el desempeño económico-financiero de las empresas}

El desempeño económico-financiero de las empresas no se explica totalmente por el aumento de la calidad y de la eficiencia de sus procesos productivos o por la diferenciación de sus productos y servicios. Además de los accionistas, la empresa necesita posicionarse adecuadamente ante sus consumidores, proveedores y gobierno, cuyas interacciones se caracterizan por relaciones de poder asimétrico y, en ocasiones, intereses contrarios; esto es, los objetivos de maximización individual 
de los intereses de cada uno de los grupos admite una vasta gama de variantes, sin - con todo - existir una que pueda maximizar todos ellos simultáneamente (Waddock y Graves, 1997).

Una modificación negativa en el desempeño económico de una empresa, por consiguiente, podrá afectar a su desempeño social y ambiental. Sin embargo, existen controversias en lo referente a la respuesta positiva de los accionistas frente a las inversiones ambientales. Existe la creencia de que esas inversiones reducirán el beneficio y no traerán la rentabilidad esperada para quien invierta. En otra visión, más social, se observa que además de que esas inversiones influyen en la eficiencia de los procesos productivos de la empresa, también refuerzan la imagen de que la empresa se preocupa por los problemas ambientales que envuelven sus procesos productivos. Además, se presupone que los inversionistas no quieren estar vinculados a una empresa que no tengan preocupación por la cuestión ecológica (Porter y Van Der Linde, 1995; Preston y O’Bannon, 1997; Griffin y Mahón, 1997; Spicer, 1978; Roman et al., 1999).

La necesidad que tienen las organizaciones de asumir una responsabilidad social, superando la obligación mínima de producir bienes y servicios para la sociedad, conduce a esas empresas a tener que integrar las variables socio ambientales en sus sistemas de gestión; así, establecen una armonía entre los propios objetivos económico-financieros y los de carácter social y ambiental, con lo que posibilitan una complementariedad entre la gestión de los resultados económicos y la RSC (Ochovo et al., 2000).

No existe consenso sobre cuál es la medida más apropiada para la medición del desempeño financiero o de la RSC; sin embargo, Roman et al. (1999) revisaron la relación entre RSC y desempeño financiero en las 62 investigaciones, producidas a lo largo de 25 años, tratadas por Griffin y Mahón (1997). Éstas trataban el mismo tema, pero con énfasis en las inconsistencias metodológicas, centrándose en la industria química y usando datos de fuentes múltiples. La contribución de Roman et al. (1999) fue recategorizar los resultados de dichas investigaciones, presentando 26 reclasificaciones del estudio. Al final, él clasificó 33 estudios sugiriendo una relación positiva entre RSC y desempeño financiero, cinco con relación negativa, catorce sin relación o relación no concluyente y el resto de las investigaciones habían sido eliminadas del estudio original por presentar medidas inválidas, ya que todas estas investigaciones estaban basadas en variables de opinión de los responsables. 
Preston y O'Bannon (1997) analizaron la relación entre indicadores de RSC y desempeño financiero de 67 grandes empresas americanas en el periodo de 1982 a 1992. El análisis longitudinal de los datos evidenció una correlación positiva entre los indicadores de desempeño social y el financiero; es decir, buenos desempeños financieros estaban presentes en empresas con un buen desempeño de RSC, un resultado congruente con la teoría de los grupos de interés. Adicionalmente, los resultados revelaron que el desempeño financiero ocurrió en la misma época en que mejoraban los aspectos de RSC.

De acuerdo con Scholtens (2008) existen dos relaciones entre desempeño financiero y RSC. Por un lado, un buen desempeño financiero está directamente relacionado con los fondos con los que realizan las inversiones que mejoran el funcionamiento ambiental y social de la empresa; por otro, un buen funcionamiento ambiental y social dará lugar a un buen desempeño financiero debido al uso eficiente de los recursos naturales y a la correcta utilización de los medios sociales en la producción.

Mahoney y Roberts (2007) detallan que los inversionistas institucionales están atentos a cómo las empresas manejan las dimensiones relacionadas con la RSC, de forma que invierten más en compañías que tienen un grado más elevado de compromiso con las causas sociales y medio ambientales.

Los estudios realizados por Porter y Van Der Linde (1995) y Spicer (1978) muestran resultados que apuntan hacia una relación positiva y significativa entre RSC y desempeño financiero. Esa relación positiva se basa en que si la empresa actúa sobre sus responsabilidades sociales y ambientales está actuando en el sentido de ganar fiabilidad en el mercado y, por otro lado, en que su desempeño socio-ambiental está directamente relacionado con una ganancia de eficiencia al evitarse el desperdicio de recursos y tratar posteriormente los residuos, lo que aumentaría el desempeño económico-financiero.

En la investigación efectuada por Al-Tuwaijri et al. (2004) fue posible afirmar que el desempeño social y ambiental y el desempeño económico están efectivamente relacionadas siguiendo una secuencia lógico-deductiva de implicación de uno en el otro. El estudio se basó en la premisa de que las estrategias corporativas, las políticas derivadas de éstas y las respectivas decisiones afectan simultáneamente al desempeño socio-ambiental y al desempeño económico de la empresa. 
Ya el estudio efectuado por Waddock y Graves (1997) reveló que los resultados sociales de la empresa son tanto una causa como una consecuencia de los resultados financieros. Así, un mejor resultado financiero lleva a un incremento de los resultados sociales. Tal hecho lleva a considerar que las empresas que invierten en las áreas social y ambiental pueden tener ventajas competitivas derivadas de este planteamiento.

Corregan y Cantú (2005) realizaron una investigación con 52 empresas mexicanas para verificar cuáles eran las principales estrategias utilizadas por esas firmas. Fue posible concluir que, a pesar de aplicar diferentes programas sociales, pocas de dichas empresas, y que contestaron la encuesta, dieron a esos programas un enfoque estratégico, de tal forma que sus desempeños financieros fueron perjudicados, lo que ocasionó la pérdida de beneficios económicos.

Aunque los estudios realizados sobre la relación entre el desempeño social de las empresas y su desempeño financiero puedan tener resultados divergentes — debido a la variedad de metodologías y parámetros adoptados, falta de rigor, por ejemplo - también demostraron que la RSC no representa una restricción a la maximización de los beneficios. Aunque su obligación sea la obtención de beneficios, las empresas pueden, a la vez, contribuir al cumplimiento de los objetivos sociales y ambientales, los cuales deben ser considerados por los inversionistas.

\section{La competitividad empresarial y la RSC}

La RSC de la empresa es un factor muy importante para que ésta pueda alcanzar ventajas competitivas relacionadas positivamente con su rentabilidad financiera. La ventaja competitiva está directamente reflejada en las capacidades de la empresa para obtener un resultado financiero superior al de sus competidores (Arend, 2003). Así, la estrategia centrada en la RSC de la empresa resuelve la tensión existente entre los objetivos sociales y de rentabilidad, puesto que requiere de ambos.

En la actualidad existe una preocupación creciente por el desarrollo sostenible, ya sea por la presión de los diferentes grupos de interés o por el mayor convencimiento empresarial de su capacidad de crear valor a largo plazo. Para muchos la posibilidad de diferenciarse de la competencia y obtener ventajas competitivas se relaciona con la RSC (Adams y Zutshi, 2004; King, 2002), lo que supone la gestión de los recursos disponibles desde este punto de vista. La RSC es vista por una serie de empresas como una estrategia de diferenciación (McWilliams y Siegel, 2001). 
De acuerdo con Epstein y Roy (2003) solamente es posible integrar aspectos sociales y ambientales en la estrategia empresarial cuando las empresas consiguen establecer una relación que justifique los negocios para un buen desempeño socioambiental. Aunque sea posible actuar de forma responsable sencillamente porque se cree que es algo que se debe hacer, tal justificación es vulnerable a los cambios en la gestión, a los ciclos financieros y ante cambios en las prioridades de los consumidores. Sin poder medir aquello que se pretende gestionar se vuelve más difícil obtener el apoyo necesario para poner en práctica los proyectos de naturaleza social y ambiental.

Sin embargo, Borger (2001) afirma que la RSC es una variable importante en la estrategia competitiva de la empresa, pues responde a cuestiones esenciales como el reto de la globalización que crea nuevas oportunidades, pero también incrementa la complejidad organizativa y la necesidad de reforzar el prestigio como elemento de la competitividad. Se entiende que las empresas que cumplan sus requisitos contribuirán al funcionamiento sostenible de los mercados comerciales.

En opinión de algunos investigadores (Barney, 1991; Hart, 1995; Orlitzky et al., 2003), las empresas que son socialmente más responsables aumentan sus ventajas competitivas, conduciendo a un desempeño financiero más satisfactorio que el de las empresas que no priorizan la RSC.

En mayo de 1999 la encuesta realizada por Millennium Poll —a 25,000 ciudadanos de 23 países en seis continentes sobre la RSC - llegó a la conclusión de que la mitad de la población de los países analizados está prestando atención al comportamiento social de las compañías, pues la percepción que se tiene de una empresa en el mundo está más directamente vinculada a su responsabilidad social (56\%) que a la calidad de su marca (40\%) o a la percepción de la gestión de su negocio (34\%) (Business Impact, 2006).

En otra encuesta realizada en 1998 por MORI Research (Business Impact, 2006) se demostró que en Reino Unido hay una gran correlación entre la responsabilidad social de una empresa y sus consumidores. En la investigación de MORI, realizada entre adultos británicos, fue posible demostrar que un 17\% habían boicoteado el producto de una compañía bajo argumentos éticos y que un 19\% habían elegido un producto/servicio debido a la reputación ética de la empresa. 
Una inversión social y ambiental bien gestionada podrá garantizar a cualquier empresa una posición predominante en la sociedad donde actúa y éste es factor decisivo en el crecimiento de la imagen de cualquier organización. Con una imagen reforzada y dependiendo de los resultados de los proyectos sociales por ella financiados, la empresa se vuelve más conocida y vende más sus productos y servicios; además, su marca gana, sobre todo, mayor visibilidad, aceptación y potencialidad.

\section{La influencia de la RSC en el comportamiento de los consumidores e inver- sores}

La adopción de estrategias relacionadas con la RSC supone un cambio cultural que repercute en las diferentes áreas organizativas de las empresas (Joyner y Payne, 2002). Se formaliza a través de políticas y sistemas de gestión en los ámbitos económico, social y medioambiental (triple bottom line) (Carroll, 1999). En este sentido, las entidades han de acomodar sus actividades, estructuras organizativas, procesos o productos de acuerdo con esta filosofía. Esta realidad hace que las inversiones, las políticas de diversificación, el desarrollo de nuevos productos y procesos, los cambios organizacionales o las políticas de personal se puedan estudiar desde el punto de vista de la sostenibilidad.

Una buena evaluación de la RSC se relaciona con una mejoría de las ventas, de la reputación de la organización y con la reducción de la probabilidad de boicots que ocurren cuando consumidores, organizaciones no gubernamentales (ONG), inversionistas o empleados se movilizan contra la compañía (Márquez y Fombrun, 2005; Gardberg y Fombrun, 2002). La reputación es un valioso activo intangible; sin embargo, el tamaño de ese efecto todavía es desconocido (Fombrun, 2001).

Dunn (1998), presidente del Business for Social Responsibility (BSR), declara que ser socialmente responsable es uno de los pilares de sustentación de los negocios, tan importante como la calidad, la tecnología y la capacidad de innovación. Así, cuando la empresa es socialmente responsable atrae a los consumidores y aumenta la cifra de ventas, generando más beneficio para los accionistas. También es una señal de reputación corporativa y añade valor a la marca.

Un estudio efectuado con 750 consumidores de cinco ciudades españolas, realizado por PricewaterhouseCoopers (2008), demostró que casi el 74\% de los encuestados afirma estar dispuesto a dejar de comprar productos a las empresas que no sean socialmente responsables. 
Las empresas dedican más recursos financieros y comerciales que nunca al patrocinio de iniciativas de RSC. Sin embargo, los consumidores e inversionistas están más atentos a las iniciativas de las empresas en relación con sus prácticas sociales y ambientales. Las iniciativas de RSC constituyen un medio innovador para fortalecer la relación con los clientes e inversores (Stainer, 2002).

\section{Metodología}

Se seleccionarán para el análisis dos empresas del ramo petrolero: Petrobrás, con sede en Brasil, y Repsol, con sede en España; ambas han sido elegidas para el estudio debido a su representatividad en el escenario brasileño, español e internacional.

El estudio pretende analizar la relación existente entre la RSC y el desempeño económico-financiero de las empresas investigadas, comprendiendo el periodo 2002 a 2007. Los datos fueron extraídos a través de las web de Petrobrás y Repsol, así como de los siguientes informes: anual consolidado, anual individual, anual de gobierno corporativo y de responsabilidad corporativa. La verificación de la afinidad existente entre la RSC y el desempeño económico-financiero no busca hacer inferencia de sus resultados para todas las compañías del sector. Para fines de análisis fue utilizada la regresión lineal múltiple.

\section{Limitaciones del método aplicado en la investigación}

El modelo utilizado, al confrontar el desempeño económico-financiero con la RSC de las dos empresas investigadas, no consideró aspectos que también ejercen una fuerte influencia en las ventas anuales y en los resultados obtenidos como procesos operacionales, estrategia comercial, política de recursos humanos y estrategia de relación con los clientes.

Otro hecho es que el estudio no consideró la consecuencia del incremento del poder de las empresas al asumir causas sociales, provenientes de las dificultades del Estado para cubrir las necesidades básicas del ciudadano. Esa situación hace que las empresas dejen de ser sólo productoras de bienes y servicios para influir y participar directamente en otras dimensiones sociales, transformándose en instituciones con gran influencia política y social. 


\section{Análisis y discusión de los resultados}

Inicialmente el estudio estaba compuesto por cuatro variables dependientes y por cinco variables independientes agrupadas en:

- Variables dependientes: ingresos por ventas y servicios (IVS); indicador de rentabilidad financiera (IRF); beneficio del ejercicio (BEJ) y fondos propios (FP).

- Variables independientes: inversión medioambiental (IMA); inversión en programas sociales y culturales (IPSC); gasto de personal (GP); planes de pensiones (PP) y gastos de formación y desarrollo (GFD). La variable explicativa inversión en programas sociales y culturales contiene los siguientes factores: educación y formación; desarrollo comunitario; integración social; salud; arte y cultura.

El análisis de correlaciones bivariadas entre las variables se completó habiendo sido verificadas con la utilización de los coeficientes de Pearson y el modelo de regresión, efectuado con los métodos de selección de variables: el paso al paso (stepwise) y el de todas las regresiones posibles (enter). Con el análisis de correlación, algunas de las variables incluidas inicialmente en la investigación fueron eliminadas del modelo; a continuación se explica qué variables fueron descartadas.

\section{Análisis de correlación}

A través del análisis de correlación por el método de Pearson se pudo concluir que, con excepción de la variable explicativa, inversiones en medio ambiente, las demás variables independientes poseen un grado muy significativo con las variables dependientes (IVS, BEJ y FP); pero la inclusión de todas las variables independientes en el modelo generó una matriz singular, además de que entre algunas variables explicativas fue posible observar una correlación altamente significativa, hecho que llevó a la necesidad de efectuar el análisis del factor de inflación de varianza (VIF) para evaluar la existencia de multicolinealidad. Como consecuencia de todo ello, se han eliminado del modelo las variables independientes GP, PP y GFD y también fue descartada la variable dependiente IRF.

Después de la aplicación del VIF, a través del valor de tolerancia y su inverso y de la matriz de colinealidad, se pudo verificar qué variables independientes debe- 
rían ser excluidas del modelo. Las variables con menores correlaciones parciales y causantes de multicolinealidad han sido retiradas del rol de variables, ajustándose nuevos modelos para cada práctica.

La correlación entre las variables, determinada para el estudio de Petrobrás y Repsol, está destacada en los cuadros 1 y 2, respectivamente. Se comprobó que las mejores correlaciones de la variable respuesta IPSC habían sido obtenidas con las variables dependientes IVS y BEJ en las dos prácticas analizadas. Los coeficientes de correlación con la variable independiente IPSC en relación con las variables independientes IVS y BEJ fueron significativas en $98 \%$ y $94 \%$ (cuadro 1), y en $94 \%$ y $95 \%$ (cuadro 2), respectivamente.

Entre las dos empresas investigadas, es conveniente resaltar que ninguna de las variables explicativas justifica la variación ocurrida en los indicadores de desempeño financiero en los años comprendidos entre 2002 y 2007. Otro hecho importante fue la débil correlación existente entre la variable independiente, inversiones en medio ambiente, con todas las variables dependientes incluidas en los modelos. 


\section{Cuadro 1}

\section{Coeficiente de correlación de Pearson (Petrobrás)}

\begin{tabular}{|c|c|c|c|c|c|c|c|c|c|c|}
\hline \multicolumn{2}{|c|}{ Variables } & \multirow{2}{*}{$\begin{array}{c}\text { IVS } \\
1\end{array}$} & \multirow{2}{*}{\begin{tabular}{|c|} 
IRF \\
-0.461 \\
0.357 \\
\end{tabular}} & \multirow{2}{*}{\begin{tabular}{|c|} 
BEJ \\
$.930(* *)$ \\
0.007 \\
\end{tabular}} & \multirow{2}{*}{\begin{tabular}{|c|}
$\mathbf{F P}$ \\
$.994(* *)$ \\
0
\end{tabular}} & \multirow{2}{*}{\begin{tabular}{|l|} 
IMA \\
0.349 \\
0.498 \\
\end{tabular}} & \multirow{2}{*}{$\begin{array}{c}\text { IPSC } \\
.985(* *) \\
0\end{array}$} & \multirow{2}{*}{$\begin{array}{c}\mathbf{G P} \\
.990(* *) \\
0\end{array}$} & \multirow{2}{*}{$\begin{array}{c}\mathbf{P P} \\
.884(*) \\
0.019\end{array}$} & \multirow{2}{*}{$\begin{array}{c}\text { GFD } \\
.974(* *) \\
0.001\end{array}$} \\
\hline IVS & \begin{tabular}{|c|} 
Coef. \\
Correlación \\
Sig. \\
(bilateral)
\end{tabular} & & & & & & & & & \\
\hline IRF & \begin{tabular}{|c|} 
Coef. \\
Correlación \\
Sig. \\
(bilateral) \\
\end{tabular} & $\begin{array}{l}-0.461 \\
0.357\end{array}$ & 1 & $\begin{array}{l}-0.139 \\
0.794\end{array}$ & $\begin{array}{l}-0.53 \\
0.28\end{array}$ & $\begin{array}{l}-0.384 \\
0.452\end{array}$ & $\begin{array}{r}-0.435 \\
0.389\end{array}$ & $\begin{array}{l}-0.531 \\
0.279\end{array}$ & $\begin{array}{c}-0.244 \\
0.641\end{array}$ & $\begin{array}{r}-0.334 \\
0.518\end{array}$ \\
\hline BEJ & \begin{tabular}{|c|} 
Coef. \\
Correlación \\
Sig. \\
(bilateral)
\end{tabular} & $\begin{array}{c}.930(* *) \\
0.007\end{array}$ & $\begin{array}{l}-0.139 \\
0.794\end{array}$ & 1 & $.889(*)$ & $\begin{array}{l}0.107 \\
0.841\end{array}$ & $.940(* *)$ & $\begin{array}{l}.890(*) \\
0.018\end{array}$ & $\begin{array}{c}.928(* *) \\
0.008\end{array}$ & $\begin{array}{c}.921(* *) \\
0.009\end{array}$ \\
\hline FP & $\begin{array}{c}\text { Coef. } \\
\text { Correlación } \\
\text { Sig. } \\
\text { (bilateral) } \\
\end{array}$ & $\begin{array}{c}.994(* *) \\
0\end{array}$ & $\begin{array}{l}-0.53 \\
0.28\end{array}$ & $\begin{array}{l}.889(*) \\
0.018\end{array}$ & 1 & $\begin{array}{l}0.416 \\
0.412\end{array}$ & $\begin{array}{c}.969(* *) \\
0.001\end{array}$ & $.992(* *)$ & $\begin{array}{c}.839(*) \\
0.037\end{array}$ & $\begin{array}{c}.968(* *) \\
0.002\end{array}$ \\
\hline IMA & \begin{tabular}{|c|} 
Coef. \\
Correlación \\
Sig. \\
(bilateral) \\
\end{tabular} & $\begin{array}{l}0.349 \\
0.498\end{array}$ & $\begin{array}{c}-0.384 \\
0.452\end{array}$ & 0.107 & 0.416 & 1 & 0.297 & 0.348 & 0.041 & 0.417 \\
\hline IPSC & \begin{tabular}{|c|} 
Coef. \\
Correlación \\
Sig. \\
(bilateral) \\
\end{tabular} & $.985(* *)$ & $\begin{array}{l}-0.435 \\
0.389\end{array}$ & $\begin{array}{c}.940(* *) \\
0.005\end{array}$ & $\begin{array}{c}.969(* *) \\
0.001\end{array}$ & $\begin{array}{l}0.297 \\
0.567\end{array}$ & 1 & $\begin{array}{c}.964(* *) \\
0.002\end{array}$ & $\begin{array}{c}.934(* *) \\
0.006\end{array}$ & $\begin{array}{c}.941(* *) \\
0.005\end{array}$ \\
\hline GP & $\begin{array}{c}\text { Coef. } \\
\text { Correlación } \\
\text { Sig. } \\
\text { (bilateral) } \\
\end{array}$ & $.990(* *)$ & $\begin{array}{l}-0.531 \\
0.279\end{array}$ & $\begin{array}{l}.890(*) \\
0.018\end{array}$ & $.992(* *)$ & 0.348 & $.964(* *)$ & 1 & $\begin{array}{l}.864(*) \\
0.026\end{array}$ & $\begin{array}{c}.966(* *) \\
0.002\end{array}$ \\
\hline PP & $\begin{array}{c}\text { Coef. } \\
\text { Correlación } \\
\text { Sig. } \\
\text { (bilateral) } \\
\end{array}$ & $\begin{array}{c}.884(*) \\
0.019\end{array}$ & $\begin{array}{c}-0.244 \\
0.641\end{array}$ & $\begin{array}{c}.928(* *) \\
0.008\end{array}$ & 0.037 & $\begin{array}{l}0.041 \\
0.938\end{array}$ & $\begin{array}{c}.934(* *) \\
0.006\end{array}$ & $\begin{array}{l}.864(*) \\
0.026\end{array}$ & 1 & $\begin{array}{c}.847(*) \\
0.033\end{array}$ \\
\hline GFD & \begin{tabular}{|c|} 
Coef. \\
Correlación \\
Sig. \\
(bilateral)
\end{tabular} & $.974(* *)$ & 0.518 & $.921(* *)$ & $.968(* *)$ & 0.411 & $.941(* *)$ & $.966(* *)$ & $.847(*)$ & 1 \\
\hline
\end{tabular}

** La correlación es significativa al nivel 0.01 (bilateral)

* La correlación es significante al nivel 0.05 (bilateral) 
Cuadro 2

Coeficiente de correlación de Pearson (Repsol)

\begin{tabular}{c|c|c|c|c|c|c|c|c|c|c}
\hline \multicolumn{2}{c|}{ Variables } & IVS & IRF & BEJ & FP & IMA & IPSC & GP & PP & GFD \\
\hline \multirow{2}{*}{ IVS } & Coef. Correlación & \multirow{2}{*}{1} & 0.418 & $.991(* *)$ & $.972(* *)$ & -0.084 & $.940(* *)$ & $.953(* *)$ & $.978(* *)$ & $.926(* *)$ \\
& Sig. (bilateral) & & 0.409 & 0 & 0.001 & 0.874 & 0.005 & 0.003 & 0.001 & 0.008 \\
\hline \multirow{2}{*}{ IRF } & Coef. Correlación & 0.418 & \multirow{2}{*}{1} & 0.521 & 0.204 & 0.661 & 0.594 & 0.482 & 0.336 & 0.584 \\
& Sig. (bilateral) & 0.409 & & 0.289 & 0.698 & 0.153 & 0.214 & 0.333 & 0.515 & 0.224 \\
\hline \multirow{2}{*}{ BEJ } & Coef. Correlación & $.991(* *)$ & 0.521 & 1 & $.941(* *)$ & -0.002 & $.952(* *)$ & $.946(* *)$ & $.952(* *)$ & $.935(* *)$ \\
& Sig. (bilateral) & 0 & 0.289 & & 0.005 & 0.996 & 0.003 & 0.004 & 0.003 & 0.006 \\
\hline \multirow{2}{*}{ FP } & Coef. Correlación & $.972(* *)$ & 0.204 & $.941(* *)$ & 1 & -0.26 & $.870(*)$ & $.903(*)$ & $.969(* *)$ & $.857(*)$ \\
& Sig. (bilateral) & 0.001 & 0.698 & 0.005 & & 0.619 & 0.024 & 0.014 & 0.001 & 0.029 \\
\hline \multirow{2}{*}{ IMA } & Coef. Correlación & -0.084 & 0.661 & -0.002 & -0.26 & 1 & 0.135 & 0.15 & -0.121 & 0.131 \\
& Sig. (bilateral) & 0.874 & 0.153 & 0.996 & 0.619 & & 0.799 & 0.777 & 0.819 & 0.804 \\
\hline \multirow{2}{*}{ IPSC } & Coef. Correlación & $.940(* *)$ & 0.594 & $.952(* *)$ & $.870(*)$ & 0.135 & 1 & $.967(* *)$ & $.950(* *)$ & $.998(* *)$ \\
& Sig. (bilateral) & 0.005 & 0.214 & 0.003 & 0.024 & 0.799 & & 0.002 & 0.004 & 0 \\
\hline \multirow{2}{*}{ GP } & Coef. Correlación & $.953(* *)$ & 0.482 & $.946(* *)$ & $.903(*)$ & 0.15 & $.967(* *)$ & 1 & $.960(* *)$ & $.960(* *)$ \\
& Sig. (bilateral) & 0.003 & 0.333 & 0.004 & 0.014 & 0.777 & 0.002 & & 0.002 & 0.002 \\
\hline \multirow{2}{*}{ PP } & Coef. Correlación & $.978(* *)$ & 0.336 & $.952(* *)$ & $.969(* *)$ & -0.121 & $.950(* *)$ & $.960(* *)$ & 1 & $.946(* *)$ \\
& Sig. (bilateral) & 0.001 & 0.515 & 0.003 & 0.001 & 0.819 & 0.004 & 0.002 & & 0.004 \\
\hline \multirow{2}{*}{ GFD } & Coef. Correlación & $.926(* *)$ & 0.584 & $.935(* *)$ & $.857(*)$ & 0.131 & $.998(* *)$ & $.960(* *)$ & $.946(* *)$ & 1 \\
& Sig. (bilateral) & 0.008 & 0.224 & 0.006 & 0.029 & 0.804 & 0 & 0.002 & 0.004 & \\
\hline
\end{tabular}

** La correlación es significativa al nivel 0.01 (bilateral)

* La correlación es significante al nivel 0.05 (bilateral)

\section{Análisis de regresión}

El análisis de regresión fue efectuado en seis pasos, incluyendo tres variables dependientes y dos variables independientes, ya que algunas de las variables habían sido excluidas de los modelos, según se explicó al hablar del coeficiente de correlación (cuadros 1 y 2). Para cada grupo de variables se han efectuado dos análisis de regresión.

En primer lugar se ha planteado la regresión entre la variable dependiente IVS con las variables independientes IMA e IPSC; a continuación, utilizándose la misma variable dependiente se plantea una regresión con la variable independiente IPSC por representar el mejor justificante de la variación para todas las variables dependientes incluidas en el modelo. Con la utilización de las mismas variables explicativas han sido efectuadas las pruebas, obedeciendo la misma orden, con las variables dependientes BEJ y FP. Los resultados de la regresión están presentados y analizados a continuación. 


\section{Modelos utilizados en el análisis de regresión}

Las hipótesis planteadas para el análisis de las regresiones en los seis modelos son:

- Ho: $\beta i=O(i=1.2) \rightarrow$ Las variables dependientes no están relacionadas con las variables explicativas (acepta la hipótesis nula y rechaza la hipótesis alternativa).

- $H a: \beta i \neq 0 ; \rightarrow$ Las variables dependientes están relacionadas con las variables explicativas (acepta la hipótesis alternativa y rechaza la hipótesis nula). Al menos uno de los coeficientes es distinto de cero.

\section{Análisis de la regresión lineal entre la variable dependiente (IVS) y las varia- bles independientes (IMA e IPSC)}

\section{Petrobrás}

En el primer modelo (cuadro 3) se ha utilizado las variables explicativas IMA e IPSC, que presentaron un coeficiente de determinación múltiple $\left(\mathrm{R}^{2}\right)$ de 0.974 ; esto indica que el $97.4 \%$ de la variación de la variable dependiente IVS se explica por la inclusión de esas variables independientes. En el test siguiente (cuadro 3) se excluye la variable independiente IMA y aparece una plusvalía de $0.04 \%$, la cual se debe al hecho de que la variable independiente IPSC tiene un mayor poder de predicción sobre las entradas de ventas y servicios ocurridos entre los años 2002 y 2007, ya que la probabilidad generada fue de 0.002 de aceptación, mientras la variable IMA tuvo una aceptación de 0.579, mayor que el valor crítico; de hecho, se concluye que IMA no es un buen estimador para la variable dependiente. 


\section{Cuadro 3}

Estadística de la regresión, coeficientes, estadístico “t” y varianza, modelos 1 y 2 (Petrobrás)

\begin{tabular}{|c|c|c|c|c|c|c|c|c|}
\hline \multirow[t]{2}{*}{ Modelo } & \multirow[t]{2}{*}{$\mathbf{R}^{2}$} & \multirow[t]{2}{*}{$\mathbf{F}$} & \multirow[t]{2}{*}{ Sig. } & \multirow[t]{2}{*}{ Variables } & $\begin{array}{c}\text { Coef. no } \\
\text { Estandariz. }\end{array}$ & $\begin{array}{c}\text { Coef. } \\
\text { Estandariz. }\end{array}$ & \multirow{2}{*}{$\begin{array}{c}\text { Estadístico } \\
\mathbf{t}\end{array}$} & \multirow[t]{2}{*}{ Significancia } \\
\hline & & & & & B & Beta & & \\
\hline \multirow{3}{*}{1} & \multirow{3}{*}{0.974} & \multirow{3}{*}{55.518} & \multirow{3}{*}{0.004} & (Constante) & 16416015.5 & - & 1.709 & 0.186 \\
\hline & & & & IMA & 11.129 & 0.061 & 0.621 & 0.579 \\
\hline & & & & IPSC & 53.047 & 0.967 & 9.858 & 0.002 \\
\hline \multirow{2}{*}{2} & \multirow{2}{*}{0.970} & \multirow{2}{*}{130.745} & \multirow{2}{*}{0.000} & (Constante) & 21936313.9 & - & 6.564 & 0.003 \\
\hline & & & & IPSC & 54.041 & 0.985 & 11.434 & 0.000 \\
\hline
\end{tabular}

Repsol

Analizando la estadística de regresión y la varianza en el caso de Repsol, se pudo observar que en el primer modelo la variable independiente IVS está explicada en un $92.9 \%$ por las variables explicativas IMA e IPSC, pero en el segundo modelo, con sólo la variable independiente IPSC, el poder de explicación de la variable dependiente sufre una disminución del 4.6\%. A pesar de esta reducción, el modelo que mejor se adecua para explicar la variación ocurrida en el total de las ventas y servicios de cada año es el segundo modelo (cuadro 4), pues, al igual que ocurrió en Petrobrás, la variable independiente IMA (cuadro 4) presentó un grado de aceptación (0.262) muy superior al valor crítico que fue de 0.05 ; así, esa variable deberá ser excluida del modelo para que se pueda aceptar la hipótesis alternativa.

\section{Cuadro 4}

Estadística de la regresión, coeficientes, estadístico " $t$ " y varianza, modelos 1 y 2 (Repsol)

\begin{tabular}{|c|c|c|c|c|c|c|c|c|}
\hline \multirow[t]{2}{*}{ Modelo } & \multirow[t]{2}{*}{$\mathbf{R}^{2}$} & \multirow[t]{2}{*}{ F } & \multirow[t]{2}{*}{ Sig. } & \multirow[t]{2}{*}{ Variables } & \begin{tabular}{|c|c} 
Coef. no \\
Estandariz.
\end{tabular} & $\begin{array}{c}\text { Coef. } \\
\text { Estandariz. }\end{array}$ & \multirow[t]{2}{*}{$\begin{array}{c}\text { Estadístico } \\
t\end{array}$} & \multirow[t]{2}{*}{ Significancia } \\
\hline & & & & & B & Beta & & \\
\hline \multirow{3}{*}{1} & \multirow{3}{*}{0.929} & \multirow{3}{*}{19.484} & \multirow{3}{*}{0.019} & (Constante) & 41732.745 & - & 8.824 & 0.003 \\
\hline & & & & IMA & -26.242 & -0.215 & -1.379 & 0.262 \\
\hline & & & & IPSC & 647.32 & 0.969 & 6.219 & 0.008 \\
\hline \multirow{2}{*}{2} & \multirow{2}{*}{0.883} & \multirow{2}{*}{30.255} & \multirow{2}{*}{0.005} & (Constante) & 35885.26 & - & 15.494 & 0.000 \\
\hline & & & & IPSC & 627.967 & 0.94 & 5.5 & 0.005 \\
\hline
\end{tabular}




\section{Análisis de la regresión lineal entre la variable dependiente (BEJ) y las varia- bles independientes (IMA e IPSC)}

\section{Petrobrás}

Observando los dos modelos de regresión lineal presentados en el cuadro 5, el poder explicativo en la variable dependiente BEJ alcanzó un $91.5 \%$ en el primer modelo y $88.3 \%$ en el segundo modelo. Cuando se excluye la variable independiente IMA para calcular el segundo modelo se observa un decremento de 3.2\% en el poder de predicción de la variable dependiente BEJ. En cuanto al grado de aceptación (0.360), generado en el tercer modelo para la variable independiente IMA, es un valor por encima del crítico, por lo que se concluye que esa variable no representa un buen parámetro para explicar la variación en los beneficios de los ejercicios de 2002 a 2007. Por eso, el cuarto modelo (cuadro 5) tiene mayor poder para predecir la variación ocurrida en la variable dependiente.

\section{Cuadro 5}

Estadística de la regresión, coeficientes, estadístico " $t$ " y varianza, modelos 3 y 4 (Petrobrás)

\begin{tabular}{|c|c|c|c|c|c|c|c|c|}
\hline \multirow[t]{2}{*}{ Modelo } & \multirow[t]{2}{*}{$\mathbf{R}^{2}$} & \multirow[t]{2}{*}{$\mathbf{F}$} & \multirow[t]{2}{*}{ Sig. } & \multirow[t]{2}{*}{ Variables } & $\begin{array}{c}\text { Coef. no } \\
\text { Estandariz. }\end{array}$ & \begin{tabular}{|c|} 
Coef. \\
Estandariz.
\end{tabular} & \multirow{2}{*}{$\begin{array}{c}\text { Estadístico } \\
\mathrm{t}\end{array}$} & \multirow[t]{2}{*}{ Significancia } \\
\hline & & & & & B & Beta & & \\
\hline \multirow{3}{*}{3} & \multirow{3}{*}{0.915} & \multirow{3}{*}{16.244} & \multirow{3}{*}{0.025} & (Constante) & 2099963.11 & - & 2.223 & 0.113 \\
\hline & & & & IMA & 3.919 & -0.19 & -1.078 & 0.360 \\
\hline & & & & IPSC & 1.176 & 0.996 & 5.664 & 0.011 \\
\hline \multirow{2}{*}{4} & \multirow{2}{*}{0.883} & \multirow{2}{*}{30.106} & \multirow{2}{*}{0.005} & (Constante) & 809946.736 & - & 3.176 & 0.034 \\
\hline & & & & IPSC & 1.145 & 0.94 & 5.487 & 0.005 \\
\hline
\end{tabular}

Repsol

En el análisis de la RSC de Repsol, con relación a los beneficios del ejercicio obtenidos entre los años de 2002 a 2007, el cuarto modelo fue el más adecuado para explicar la variación ocurrida en la variable dependiente para ese mismo periodo, aunque la diferencia presentada entre los dos modelos para el coeficiente de determinación $\left(\mathrm{R}^{2}\right)$ haya sido tan sólo de $1.7 \%$. El coeficiente estandarizado para la variable IMA, incluida en el tercer modelo, fue negativo y presentó un grado de aceptación mayor de 5\%, lo que significa que esa variable no representa un buen parámetro de predicción para la variable dependiente BEJ. Para que la hipótesis 
alternativa se acepte, se concluye que el mejor modelo para predecir la variable dependiente es el modelo 4, cuyo valor de aceptación, tanto de la ecuación (0.003) como de la variable independiente IPSC (0.003), está por debajo del valor crítico que es de 0.05 .

\section{Cuadro 6}

Estadística de la regresión, coeficientes, estadístico “t” y varianza, modelos 3 y 4 (Repsol)

\begin{tabular}{|c|c|c|c|c|c|c|c|c|}
\hline Modelo & $\mathbf{R}^{2}$ & $\mathbf{F}$ & Sig. & Variables & $\begin{array}{c}\begin{array}{c}\text { Coef. no } \\
\text { Estandariz. }\end{array} \\
\text { B }\end{array}$ & $\begin{array}{c}\begin{array}{c}\text { Coef. } \\
\text { Estandariz. }\end{array} \\
\text { Beta }\end{array}$ & $\begin{array}{c}\text { Estadístico } \\
\mathbf{t}\end{array}$ & Significancia \\
\hline \multirow{3}{*}{3} & \multirow{3}{*}{0.923} & \multirow{3}{*}{18.060} & \multirow{3}{*}{0.021} & (Constante) & 2442.906 & - & 8.426 & 0.004 \\
\hline & & & & IMA & -0.964 & -0.133 & -0.826 & 0.469 \\
\hline & & & & IPSC & 38.35 & 0.97 & 6.01 & 0.009 \\
\hline \multirow{2}{*}{4} & \multirow{2}{*}{0.906} & \multirow{2}{*}{38.500} & \multirow{2}{*}{0.003} & (Constante) & 2228.205 & - & 18.106 & 0.000 \\
\hline & & & & IPSC & 37.64 & 0.952 & 6.205 & 0.003 \\
\hline
\end{tabular}

\section{Análisis de la regresión lineal entre la variable dependiente (FP) y las varia- bles independientes (IMA e IPSC)}

\section{Petrobrás}

El coeficiente de determinación múltiple $\left(\mathrm{R}^{2}\right)$, que representa la proporción de la variación en FP (cuadro 7) que es explicada a través del conjunto de variables predictivas seleccionadas, presentó un valor igual 0.957 (modelo 5) y 0.939 (modelo 6); es decir, $95.7 \%$ y $93.9 \%$, respectivamente, de la variación en el valor de los fondos propios acumulados entre los años 2002 y 2007.

En el sexto modelo (cuadro 7) fue excluida la variable IMA, y aunque aparezca decrecimiento de $1.8 \%$ en el poder explicativo de la variable dependiente, ésta resulta ser la mejor ecuación, ya que la variable IMA no es un buen factor de explicación para la variación en fondos propios al sobrepasar su valor de aceptación (0.127) al valor crítico adoptado. 


\section{Cuadro 7}

Estadística de la regresión, coeficientes, estadístico “t”" y varianza, modelos 5 y 6 (Petrobrás)

\begin{tabular}{|c|c|c|c|c|c|c|c|c|}
\hline \multirow[t]{2}{*}{ Modelo } & \multirow[t]{2}{*}{$\mathbf{R}^{2}$} & \multirow[t]{2}{*}{$\mathbf{F}$} & \multirow[t]{2}{*}{ Sig. } & \multirow[t]{2}{*}{ Variables } & $\begin{array}{c}\text { Coef. no } \\
\text { Estandariz. }\end{array}$ & $\begin{array}{c}\text { Coef. } \\
\text { Estandariz. }\end{array}$ & \multirow{2}{*}{$\begin{array}{l}\text { Estadís- } \\
\text { tico t }\end{array}$} & \multirow[t]{2}{*}{ Significancia } \\
\hline & & & & & B & Beta & & \\
\hline \multirow{3}{*}{5} & \multirow{3}{*}{0.957} & \multirow{3}{*}{33.063} & \multirow{3}{*}{0.009} & (Constante) & -1782084.28 & - & -0.245 & 0.822 \\
\hline & & & & IMA & 15.091 & 0.14 & 1.112 & 0.347 \\
\hline & & & & IPSC & 29.972 & 0.927 & 7.36 & 0.005 \\
\hline \multirow{2}{*}{6} & \multirow{2}{*}{0.939} & \multirow{2}{*}{61.262} & \multirow{2}{*}{0.001} & (Constante) & 5703667.76 & - & 2.016 & 0.114 \\
\hline & & & & IPSC & 31.319 & 0.969 & 7.827 & 0.001 \\
\hline
\end{tabular}

Repsol

El mismo procedimiento fue realizado para el grupo de datos extraído de los informes de Repsol, conforme a los valores indicados en el cuadro 8. En los ajustes de los modelos 5 y 6 de la regresión para los datos observados se verificó que la variable independiente IMA presenta un valor de aceptación mayor que lo permitido (0.05), por lo que fue excluida del modelo 5. Así, aplicamos el modelo 6 sólo con la variable independiente IPSC, que generó un coeficiente de determinación de 0.756 . Se concluye que la variable dependiente Fondos Propios se explica en un $75.6 \%$ por la variable independiente IPSC.

\section{Cuadro 8}

Estadística de la regresión, coeficientes, estadístico “t”" y varianza, modelos 5 y 6 (REPSOL)

\begin{tabular}{|c|c|c|c|c|c|c|c|c|}
\hline \multirow[t]{2}{*}{ Modelo } & \multirow[t]{2}{*}{$\mathbf{R}^{2}$} & \multirow[t]{2}{*}{$\mathbf{F}$} & \multirow[t]{2}{*}{ Sig. } & \multirow[t]{2}{*}{ Variables } & $\begin{array}{c}\text { Coef. no } \\
\text { Estandariz. }\end{array}$ & $\begin{array}{c}\text { Coef. } \\
\text { Estandariz. }\end{array}$ & \multirow{2}{*}{$\begin{array}{c}\text { Estadístico } \\
\mathbf{t}\end{array}$} & \multirow[t]{2}{*}{ Significancia } \\
\hline & & & & & B & Beta & & \\
\hline \multirow{3}{*}{5} & \multirow{3}{*}{0.901} & \multirow{3}{*}{13.683} & \multirow{3}{*}{0.03} & (Constante) & 16056.587 & - & 10.063 & 0.002 \\
\hline & & & & IMA & -13.479 & -0.384 & -2.099 & 0.127 \\
\hline & & & & IPSC & 176.68 & 0.921 & 5.031 & 0.015 \\
\hline \multirow{2}{*}{6} & \multirow{2}{*}{0.756} & \multirow{2}{*}{12.401} & \multirow{2}{*}{0.02} & (Constante) & 13053.047 & - & 13.589 & 0.000 \\
\hline & & & & IPSC & 166.74 & 0.87 & 3.522 & 0.024 \\
\hline
\end{tabular}




\section{Resumen de la regresión. Ecuación y análisis gráfico}

Las ecuaciones de regresión lineal (cuadros 9, 10, 11, 12, 13 y 14) que describen la relación entre el valor de las ventas y servicios y las variables independientes (modelo 1 y 2) en el periodo 2002 a 2007 son:

\section{Cuadro 9}

Ecuaciones de la regresión, modelos 1 y 2 (Petrobrás)

\begin{tabular}{l}
\hline \multicolumn{1}{c}{ Ecuaciones $=$ Ingresos por ventas y servicios $($ cuadro 3) } \\
\hline IVS $=16416015.5+11.129$ (IMA) +53.047 (IPSC) [modelo 1] \\
\hline IVS $=21936313.9+54.041$ (IPSC) [modelo 2] \\
\hline
\end{tabular}

\section{Cuadro 10}

Ecuaciones de la regresión, modelos 1 y 2 (Repsol)

\begin{tabular}{l}
\hline \multicolumn{1}{c}{ Ecuaciones $=$ Ingresos por ventas y servicios $($ cuadro 4) } \\
\hline IVS $=41732.745-26.242($ IMA $)+647.32$ (IPSC) [modelo 1] \\
\hline IVS $=35885.26+627.967($ IPSC) [modelo 2] \\
\hline
\end{tabular}

\section{Cuadro 11}

Ecuaciones de la regresión, modelos 3 y 4 (Petrobrás)

\begin{tabular}{c} 
Ecuaciones $=$ Beneficio del ejercicio $($ cuadro5) \\
\hline $\mathrm{BEJ}=4667981.4-4.225(\mathrm{IMA})+6.662(\mathrm{IPSC})[$ modelo 3] \\
\hline $\mathrm{BEJ}=2572379+6.285(\mathrm{IPSC})[$ modelo 4] \\
\hline
\end{tabular}

\section{Cuadro 12}

Ecuaciones de la regresión, modelos 3 y 4 (Repsol)

\begin{tabular}{l}
\hline Ecuaciones $=$ Beneficio del ejercicio $($ cuadro 6) \\
\hline $\mathrm{BEJ}=2442.906-0.964(\mathrm{IMA})+38.35(\mathrm{IPSC})[$ modelo 3] \\
\hline $\mathrm{BEJ}=2228.205+37.64(\mathrm{IPSC})[$ modelo 4] \\
\hline
\end{tabular}




\section{Cuadro 13}

Ecuaciones de la regresión, modelos 5 y 6 (Petrobrás)

\begin{tabular}{c} 
Ecuaciones $=$ Fondos propios $($ cuadro7 $)$ \\
\hline $\mathrm{FP}=-1782084.28+15.091(\mathrm{IMA})+29.972(\mathrm{IPSC})[$ modelo 5$]$ \\
\hline $\mathrm{FP}=5703667.76+31.319(\mathrm{IPSC})[$ modelo 6] \\
\hline
\end{tabular}

\section{Cuadro 14}

Ecuaciones de la regresión, modelos 5 y 6 (Repsol)

\begin{tabular}{l} 
Ecuaciones $=$ Fondos propios $($ cuadro 8) \\
\hline IVS $=16056.587-13.479($ IMA $)+176.68($ IPSC $)[$ modelo 5] \\
\hline IVS $=13053.047+166.74($ IPSC) $[$ modelo 6] \\
\hline
\end{tabular}

En el análisis del " $t$ " estadístico de las ecuaciones donde se han considerado las variables IMA e IPSC es posible concluir que la hipótesis de no haber regresión (Ho) debe ser aceptada; es decir, en los modelos 1, 3 y 5 de las regresiones aplicadas para las empresas Petrobrás y Repsol el valor de "t" para esos datos es, respectivamente, 0.621 y -1.379 (modelo 1, cuadros 3 y 4), 1.078 y 0.826 (modelo 3, cuadros 4 y 5), 1.112 y -2.099 (modelo 5, cuadros 7 y 8), para un grado de libertad de tres (figura 1) y con valor crítico de 3.18 (tabla de distribución, Levine et al. 1998). Para todos los modelos que incluyen únicamente la variable explicativa IPSC debe aceptarse la hipótesis alternativa y rechazar la hipótesis nula conforme los "t" estadísticos constantes en los cuadros 3, 4, 5, 6, 7 y 8, cuyos valores están todos fuera del margen crítico de 2.78 (figura 2). 
Figura 1

Gráfica de la distribución “t”" para los datos de los modelos 1, 3 y 5

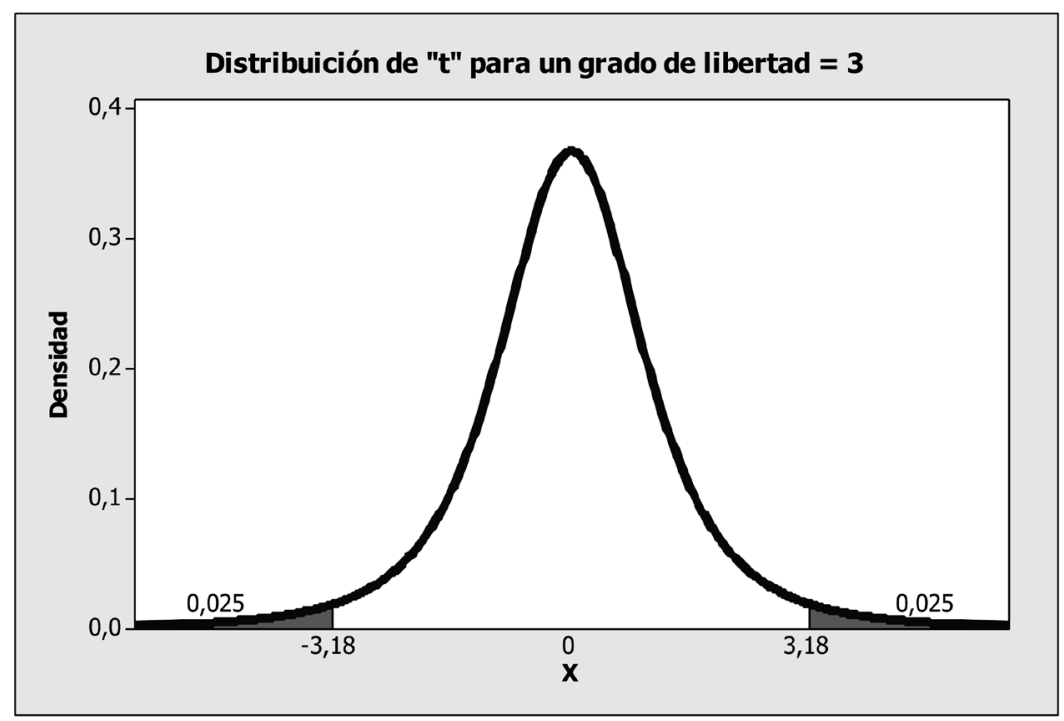

Figura 2

Gráfica de la distribución “t”" para los datos de los modelos 2, 4 y 6

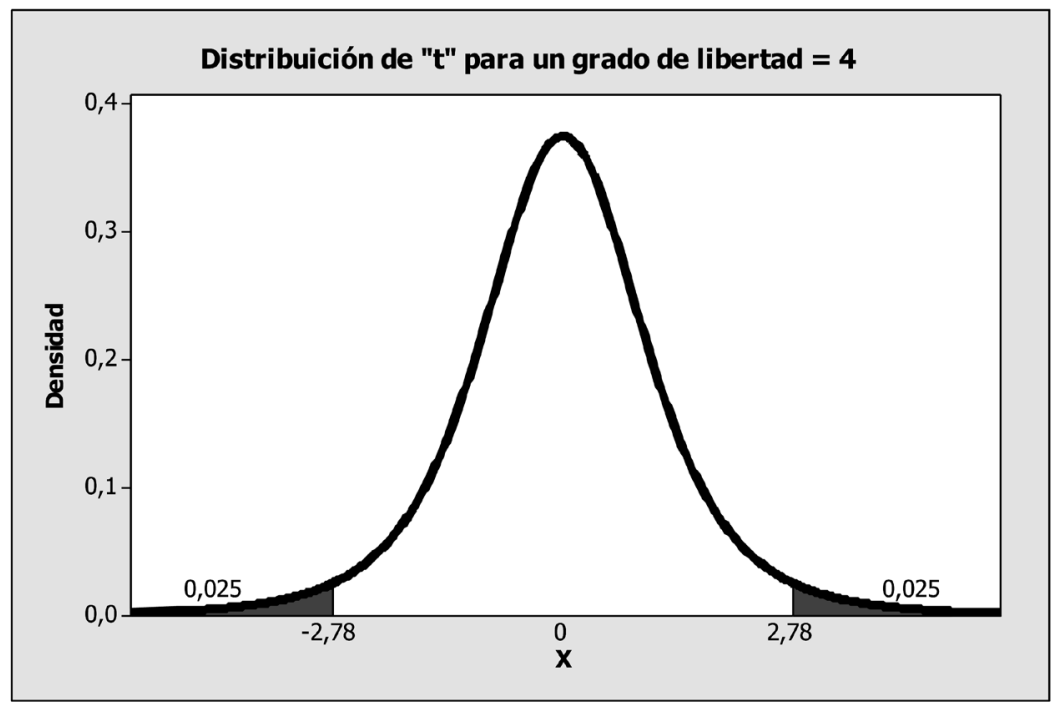


En el análisis de la varianza también es posible comprobar que debemos aceptar la regresión únicamente para los modelos 2, 4 y 6 que incluyen tan sólo la variable explicativa IPSC cuyos valores de "F" están todos fuera del valor crítico de 7.71 (figura 3) y 9.55 (figura 4) para los grados de libertad numerador=1 y denominador $=4$, y para numerador $=2$ y denominador $=3$, respectivamente, conforme a las tablas de la distribución de "F" (Levine et al. 1998).

Figura 3

Gráfica de distribución "F" para los datos de los modelos 1, 3 y 5

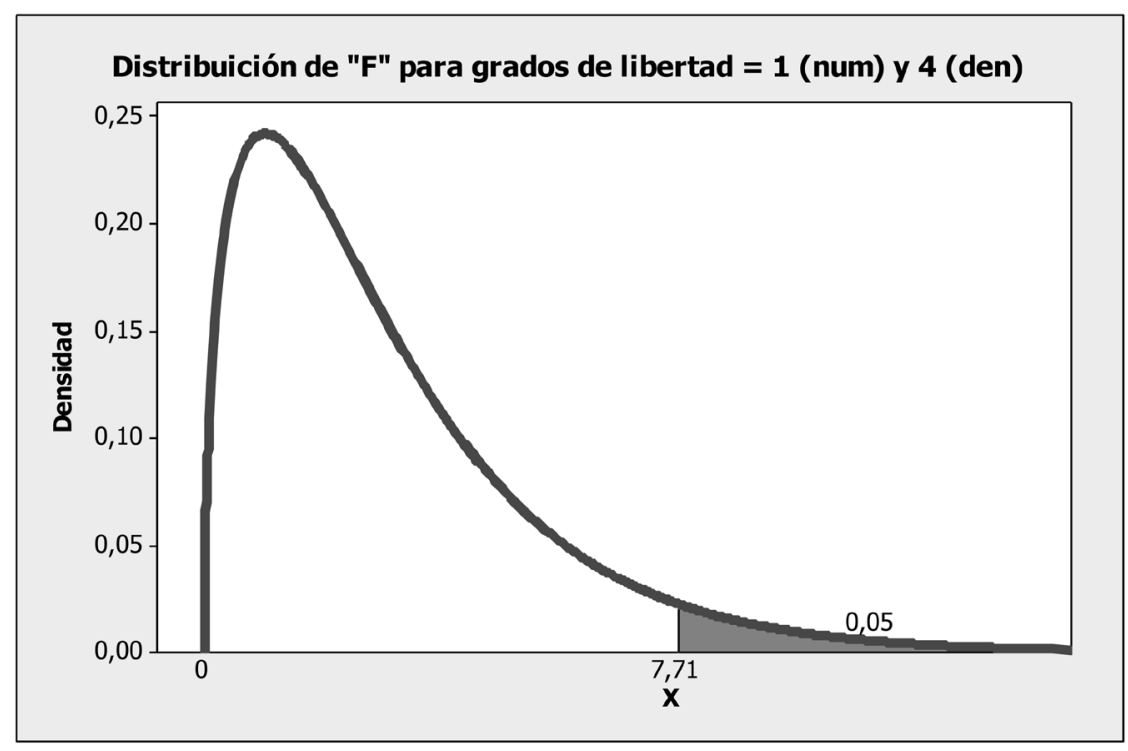


Figura 4

Gráfica de distribución "F" para los datos de los modelos 2, 4 y 6

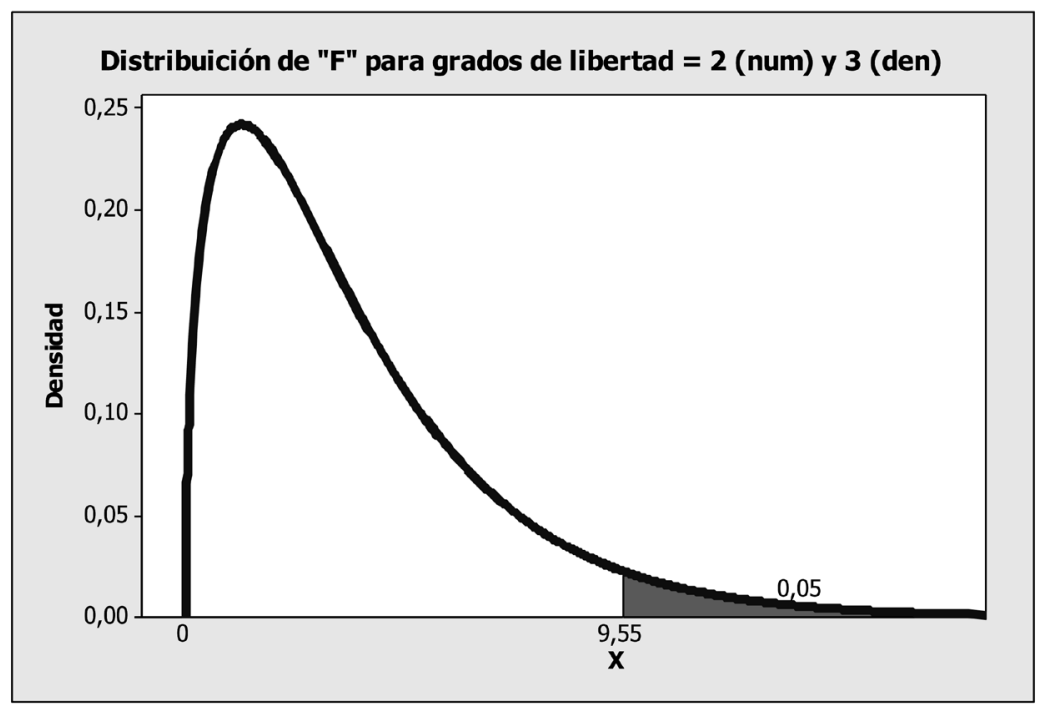

\section{Análisis gráfico de las inversiones en RSC de Petrobrás y Repsol}

La mayor inversión en RSC de Petrobrás, entre los años 2002 y 2007, fue en el último año analizado, donde el valor invertido en responsabilidad social y ambiental alcanzó la cifra de 5.22 mil millones de reales, el equivalente a 2 mil millones de euros. El valor de la inversión en RSC en el año es equivalente al 6.24\% (figura 5) de las ventas totales en este mismo año, representada por el valor de $218.2 \mathrm{mil} \mathrm{mi-}$ llones de reales (83.67 mil millones de euros). En 2007, el beneficio del ejercicio alcanzó 21.5 mil millones de reales, el equivalente a 8.2 mil millones de euros. ${ }^{1}$

${ }^{1}$ Para fines de conversión de los valores en reales para euros se utilizaron las tasas de cambio presentadas en la web del Banco Central de Brasil (2008) el 31 de diciembre de cada año. 
Figura 5

Gráfica de porcentaje anual de inversión en RSC en relación con sus ingresos anuales para Petrobrás

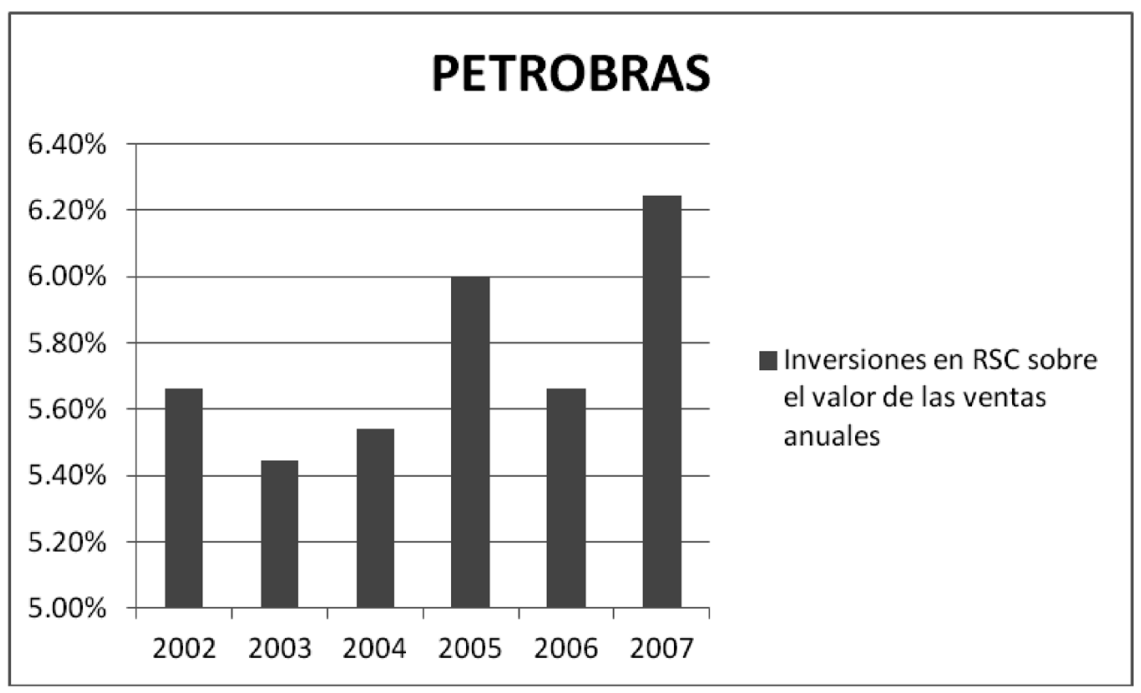

Aunque Repsol ha venido desarrollando desde hace más tiempo sus actividades sociales y ambientales, fue a partir de 2004 cuando comenzó a divulgar con más detalle sus informes de RSC. En los años 2002 y 2003 los datos financieros presentados en los informes de RSC enfatizaban más las actividades relacionadas con el medioambiente, mientras que las relacionadas con las realizaciones sociales eran presentadas, en gran parte, sólo en indicadores y ratios.

En 2004 Repsol aplicó 523 millones de euros a RSC: un 1.30\% (figura 6) sobre el total de la ventas de ese año, en el que obtuvo un beneficio del ejercicio de 2.6 millones de euros. 
Figura 6

Gráfica de porcentaje anual de inversión en RSC

en relación con sus ingresos anuales para Repsol

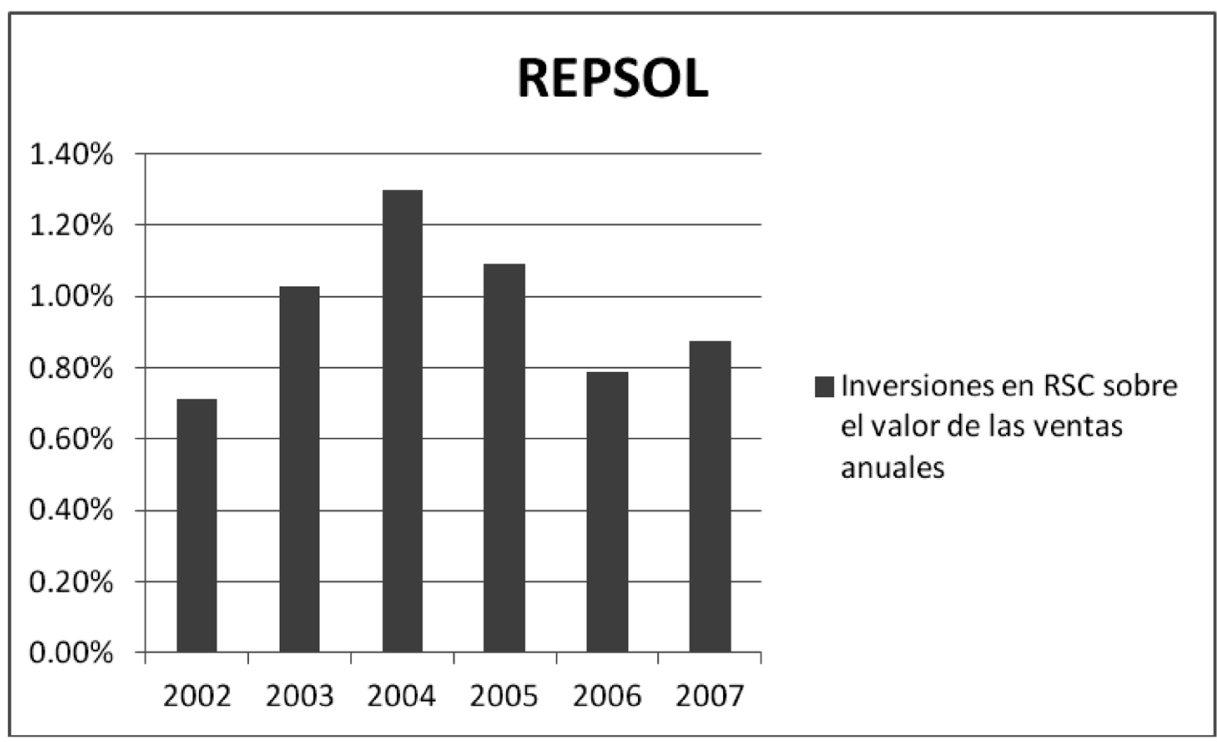

Las rentabilidades financieras se han calculado a partir de la relación entre los fondos propios y los beneficios de los ejercicios 2002 a 2007, obtenidos en los informes anuales de las dos empresas investigadas. Con esos indicadores se ha podido verificar que los mejores índices de rentabilidad financiera para Petrobrás ocurrieron en 2003 los cuales correspondieron a un 36.04\% (figura 7), mientras que para Repsol fueron en 2004, correspondiente a un 19.39\% (figura 8).

Los beneficios más altos de los ejercicios de esas dos empresas ocurrieron en 2006 para Petrobrás en un valor equivalente a 9.19 mil millones de euros y en 2007 para Repsol con un valor de 3.35 millones de euros. Los mayores valores de inversiones en RSC ya ocurrieron en 2007 para Petrobrás, con 5.22 mil millones de euros, y en 2005 para Repsol, con 556.7 millones de euros. Esos resultados demuestran que la rentabilidad financiera de esas empresas no está directamente relacionada con sus inversiones en RSC; por lo tanto, las mejores rentabilidades financieras para Petrobrás y Repsol ocurrieron en 2003 y 2004, respectivamente; en tanto que las mayores inversiones en RSC para esas empresas fueron en 2007 para Petrobrás y 2005 para Repsol, resultados que han sido posibles comprobar con los modelos 
estadísticos en los cuales la variable independiente Rentabilidad Financiera (RF) fue excluida de las ecuaciones.

Figura 7

Gráfica de la rentabilidad financiera anual (Petrobrás)

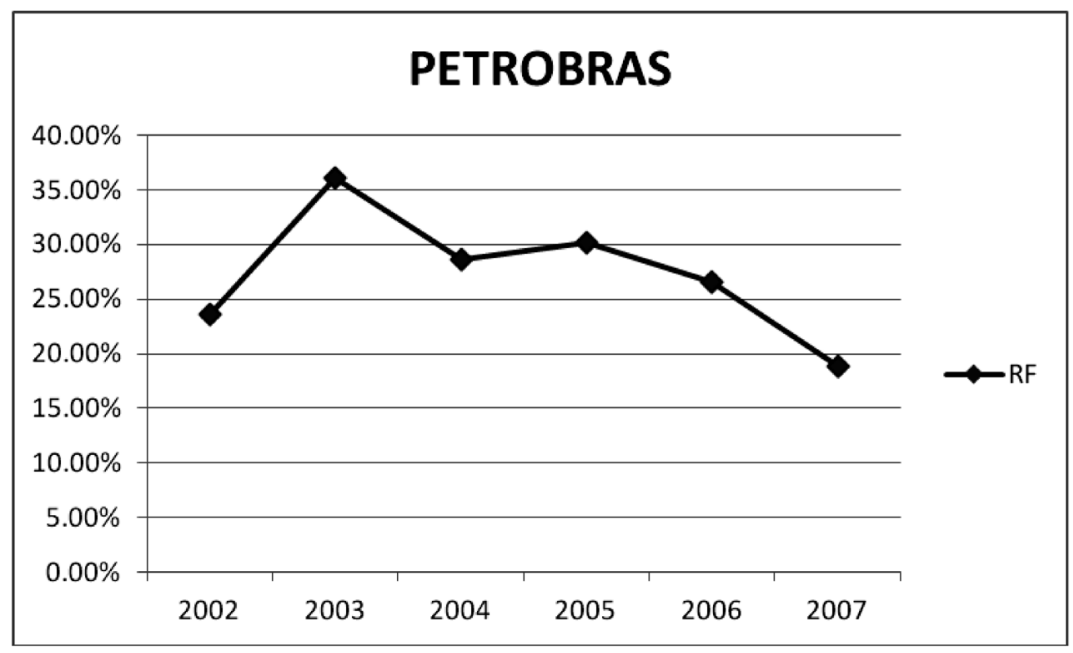

Figura 8

Gráfica de la rentabilidad financiera anual (Repsol)

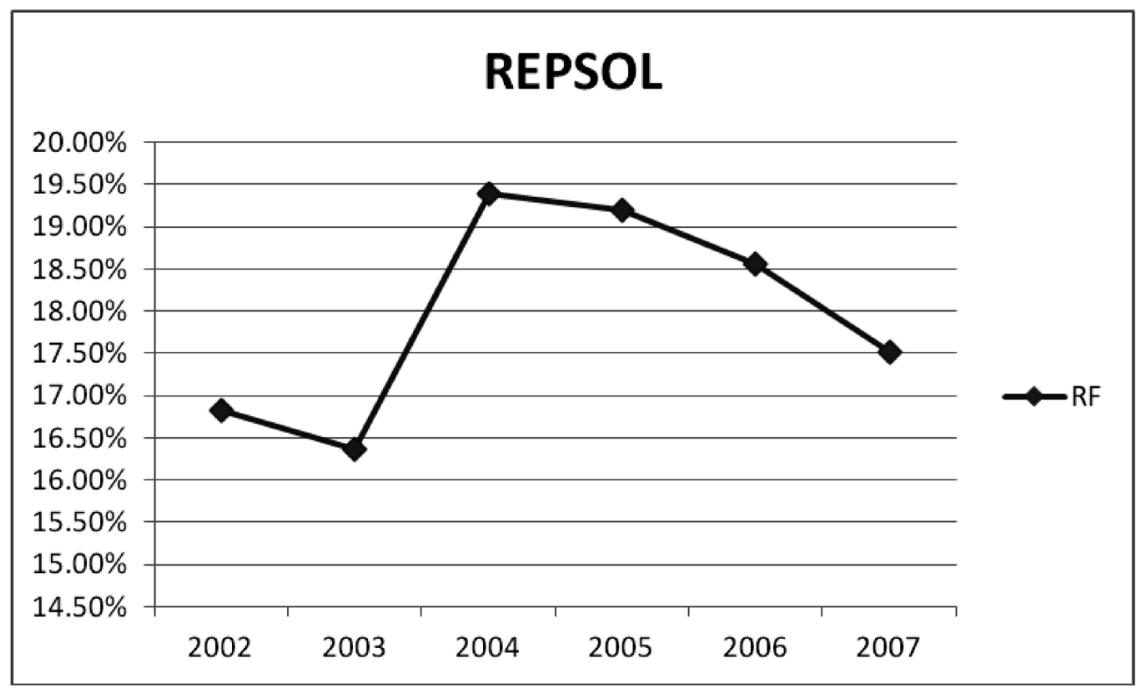




\section{Conclusiones}

Este trabajo tuvo como objetivo principal la utilización de técnicas estadísticas para analizar la relación entre el desempeño económico-financiero y la RSC de Petrobrás, S.A. y de Repsol, S.A. en el periodo 2002-2007.

En la actualidad, una parte significativa de la sociedad percibe a las empresas como organizaciones sociales centradas, de forma excesiva, en la maximización de beneficios. Esta percepción se ve reforzada por el hecho de que, en ocasiones, los mismos directivos que incurren en conductas reprobadas por la sociedad se comprometen públicamente con el bien común a través del proyecto colectivo de la responsabilidad social y ambiental.

En los análisis efectuados, los coeficientes estandarizados (Beta), en todos los modelos mostrados en los cuadros 3 a 8, apuntan a la variable inversiones en programas sociales y culturales (IPSC) como la que mejor explica sobre las variaciones ocurridas en las variables dependientes: ingresos por ventas y servicios (IVS); beneficio del ejercicio (BEJ) y fondos propios (FP).

La variable inversiones en medioambiente (IMA), por tener los mayores valores a lo largo de los años 2002 a 2007, parecía al principio ser un buen indicador para explicar la variación ocurrida en las variables dependientes al tratarse de un factor que actualmente es observado por todos los grupos de interés, pero sorprendentemente, conforme a los modelos 2,4 y 6 (cuadros 3 y 4,5 y 6 , y 7 y 8 , respectivamente), tanto en las ecuaciones calculadas con los datos obtenidos de los informes de Petrobrás como en los obtenidos de los informes de Repsol los datos apuntan a que las inversiones en programas sociales y culturales (compuesto por educación y formación; desarrollo comunitario; integración social; salud; arte y cultura) son los mejores factores de explicación para los desempeños económicos-financieros de esas dos empresas.

\section{Referencias}

Adams, C.A. y A. Zutshi (2004). Corporate Social Responsibility: Why Business Should Act Responsibly and Be Accountable. Australian Accounting Review 14 (3): 31-39. 
Al-Tuwaijri, S. A. et al. (2004). The relations among environmental disclosure, environmental performance and economic performance: a simultaneous equations approach. Accounting Organizations and Society 29: 447-471.

Arend, R. J. (2003). Revisiting the logical and research considerations of competitive advantage. Strategic Management Journal 24 (3): 279-284.

Barney, J. (1991). Firm Resources and Sustained Competitive Advantage. Journal of Management 17 (1): 99-120.

Borger, F.G. (2001). Responsabilidade Social: Efeitos da Atuação Social na Dinâmica Empresarial. Tese de Doutorado, São Paulo: FEA/USP.

Bolsa de Valores de São Paulo (2005). ISE-Índice de Sustentabilidade Empresarial. Disponible en: http://www.bovespa.com.br/indexSP.asp

Business Impact (2006) Report: Winning with Integrity. Disponible en: http://www.bitc.org.uk/applications/site_search/ ?term=\%22Winning+with+Integrity $\% 22 \&$ submit.x=9\&submit.y=14\&old term=Winning+with+Integrity\&old_term=Winning+with+Integrity\&old_ instance_id $=158312$

Carroll, A. B. (1999). Corporate Social Responsibility: Evolution of a Definitional Construct. Business and Society 38: 268-295.

Corregan, B.W.H. y J.J.S. Cantú (2005). Un estudio exploratorio sobre la estrategia social de empresas grandes ubicadas en México. Revista Contaduría y Administración enero-abril (215): 9-23.

Comissão de Valores Mobiliários (2008). Companhias Abertas-Informações periódicas e eventuais. Disponible en: http://www.cvm.gov.br/

Dunn, R. (1998). Quer uma vantagem competitiva? Revista Exame 32 (18): 5.

Epstein, M.J. y M.J. Roy (2003). Making the business case for sustainability. linking social and environmental actions to financial performance. Journal of Corporate Citizenship 9: 79-96. 
Instituto Ethos de Empresas e Responsabilidade Social, Ethos (2007). Guia para Elaboração de Balanço Social e Relatório de Sustentabilidade. Disponible en: http://www.ethos.org.br/DesktopDefault.aspx?Alias=Ethos\&Lang=pt-BR

Fernández Izquierdo, M.A. et al. (2005). La Responsabilidad Social Corporativa: relaciones entre la performance social, financiera y bursátil de la empresa, Documento de trabajo $n^{\circ} 23$, Universitat Jaume I. Castellón.

Fombrun, C. J. (2001). Corporate reputation as economic assets. The Blackwell Handbook of Strategic Management. M.A. Hitt, R.E. Freeman and J.S. Harrison (eds.), Malden: Blackwell Publishers.

Fundación Ecología y Desarrollo (2006). Anuario sobre Responsabilidad Social Empresarial en España. Santander Ediciones.

Gardberg, N.A. y Ch.J. Fombrun (2002). For better or worse-the most visible American corporate reputations. Corporate Reputation Review invierno 4 (4): $385-387$.

González, M. C. (2004). El porqué de la responsabilidad social corporativa. Boletín Económico de ICE (2813): 45-58.

Global Reporting Initiative (2008). G3 Guidelines-GRI Sustainability Reporting Framework. Disponible en: http://www.globalreporting.org/ReportingFramework/G3Guidelines/

Griffin, J.J. y J.F. Mahon (1997). The corporate social performance and corporate financial performance debate: Twenty-five years of incomparable research. Business and Society 36 (1): 5-31.

Hart, S. L. (1995). A Natural-Resource-Based View of the Firm. Academy of Management Review 20 (4): 986-1014.

Instituto Brasileiro de Análises Sociais e Econômicas, IBASE (2008). Trajetória cidadã: Betinho e a luta. Disponible en: http://www.ibase.br/modules. php?name $=$ Conteudo\&pid $=40$ 
Joyner, B.E. y D. Payne (2002). Evolution and implementation: A study of values, business ethics, and corporate social responsibility. Journal of Business Ethics 41: 297-311.

King, A. (2002). How to Gert Started in Corporate Social Responsibility. Financial Management, October

Knox, S. et al. (2005). Corporate Social Resonsability: Exploring Stakeholder Relationships and Programme Reporting across Leadign FTSE Companies. Journal of Business Ethics 61: 7-28.

Levine, D.M. et al. (1998). Statistic for Managers Using Microsoft Excel. New York: Prentice Hall.

Lewis, S. (2003). Reputation and corporate responsibilit. Journal of Communication Management 7 (4): 356-364.

Mahoney, L. y R.W. Roberts (2007). Corporate social performance, financial performance and institutional ownership in Canadian firms. Accounting Forum 31: 233-253.

Márquez, A. y C.J. Fombrun (2005). Measuring Corporate Social Responsibility. Corporate Reputation Review 7 (4): 304-308.

McWilliams, A. y D. Siegel (2001). Corporate Social Responsibility: A Theory of the Firm Perspective. Academy of Management Review 26 (1): 117-127.

Milani Filho, M. A. F. (2008). Responsabilidade social e investimento social privado: entre o discurso e a evidenciação. Revista Contabilidade e Finanças 19 (47): 89-101.

Moneva, J. M. (2005). Información sobre Responsabilidad Social Corporativa: Situación y Tendencias. Revista Asturiana de Economía (34): 43-67.

y F. Llena (1996). Análisis de la Información sobe Responsabilidad Social en las Empresas Industriales que cotizan en Bolsa. Revista Española de Financiación y Contabilidad abril-junio XXV (87): 361-402. 
Morimoto, R. et al. (2005). Corporate Social Responsibility Audit: From Theory to Practice. Journal of Business Ethics 62: 315-325.

Ochovo, R. B., D.N. Peña y A.T. Ponce (2000). El cuadro de mando como instrumento de control en la gestión social: recursos humanos y medio ambiente. Revista Española de Financiación y Contabilidad enero-marzo XXDI (103): 107-147.

Orlitzky, M. et al. (2003). Corporate social and financial performance: A metaanalysis. Organization Studies 24 (3): 403-441.

Payne, D. M. y C.A. Raiborn (2001). Sustainable Development-The Ethics Suport the Economics. Journal of Business Ethics 32: 157-168.

Petrobrás (2008). Histórico de la empresa. Disponible en:

http://www2.petrobrás.com.br/portal/frame_ri.asp?pagina=/ri/port/index. asp\&lang $=$ pt\&area $=$ ri

Porter, M. y C. Van Der Linde (1995). Green and competitive: Ending the stalemate. Harvard Business Review 73 (5): 120-134.

Preston, L. E. y D.P. O'Bannon (1997). The Corporate Social-financial performance relationship. Business and Society 36 (4): 419-429.

Pricewaterhousecoopers (2008). La actitud del consumidor hacia la Responsabilidad Social Corporativa (RSC). Disponible en: http://kc3.pwc.es/local/ es/kc3/publicaciones.nsf/V1/8FB3CF43A7F5594DC1257141002E80BF/ \$FILE/Inf_RSC_alimentacion_bebidas.pdf

Repsol (2008). Histórico de la Empresa. Disponible en: http://www.repsol.com/ es_es/todo_sobre_repsol_ypf/conocer_repsol_ypf/presentacion/

Roman, R. M. et al. (1999). The relationship between social and financial performance. Business and Society 38 (1): 109-125.

Ruf, B.M. et al. (2001). An empirical investigation of the relationship between Change in Corporate Social Performance and Financial Performance: A Stakeholder Theory Perspective. Journal of Business Ethics 32: 143-156. 
Sánchez, D.F.G. (2004). La responsabilidad social corporativa en materia ambiental Estado de la cuestión. Boletín Económico de ICE (2824): 27-43.

Scholtens, B. A. (2008). Note on the interaction between corporate social responsibility and financial performance, Ecological Economics doi:10.1016/ j.ecolecon.2008.01.024: 1-10.

Seara, L. G. (2004). La responsabilidad social de la empresa. Revista del Ministerio de Trabajo y Asuntos Sociales (50): 13-20.

Sustainable Investment Research International, SIRI (2007). Green, social and ethical funds in Europe. 2007 Review, Milan. Disponible en: www.eurosif. org/content/download/909/5094/version/1/file/Green+social+and+ethical+f unds+in+Europe_2007+Review.pdf

Spicer, B. (1978). Investors, corporate social performance and information disclosure: An empirical study. The Accounting Review 53: 94-111.

Stainer, A. (2002). Business performance \& corporate social responsibility. Management Services 46 (7): 36

Stanwick, P.A. y S.D. Stanwick (1998). The Relationship Between Corporate Social Performance, and Organizational Size, Financial Performance, and Environmental Performance: An Empirical Examination. Journal of Business Ethics 17: 195-204.

Tachizawa, T. (2004). Gestão ambiental e responsabilidade social corporativa: estratégias de negócios focadas na realidade brasileira, 2a. ed., São Paulo: Atlas.

Toro, D. (2006). El enfoque estratégico de la responsabilidad social corporativa: revisión de la literatura académica. Intangible Capital octubre-diciembre 2 (14): 338-358.

Waddock, S. y S. Graves (1997). The corporate social performance-financial performance link. Strategic Management Journal 18 (4): 303-319. 
White, A. y M. Kiernan (2004). Corporate Environmental Governance: a study into the influence of Environmental Governance and Financial Performance full report. Bristol: Environmental Agency UK. Disponible en: www.innovestgroup.com/pdfs/2004-11-09-Environmental_Governance.pdf

Zhang, R. y D. Stern (2007). Firms' Environmental and Financial Performance: An Empirical Study. The Fondazione Eni Enrico Mattei Series Index: Disponible en: http://www.feem.it/Feem/Pub/Publications/CSRPapers/default.htm 
